\title{
Um editor inteligente para assistir estudantes na tradução de problemas algébricos
}

\author{
Pedro Henrique Lemos ${ }^{1}$, Patricia Jaques ${ }^{1}$ \\ ${ }^{1}$ Programa Interdisciplinar de Pós-Graduação em Computação Aplicada (PIPCA) \\ Universidade do Vale do Rio dos Sinos (UNISINOS) \\ phl.sl@terra.com.br,patricia.jaques@gmail.com
}

\begin{abstract}
Resumo. Esse artigo descreve um editor inteligente que assiste os estudantes na tradução de enunciados de problemas em equações algébricos. $O$ editor apresenta funcionalidades que auxiliam o estudante a identificar as principais partes do problema e definir relaçõ es e equaçõ es, além de permitir a consulta de problemas relacionados que foram previamente resolvidos. Ele usa um algoritmo baseado em hipergrafos para representar os dados do problema e corrigir automaticamente a solução do estudante. Para avaliar o quanto a ferramenta contribuiu ao desempenho dos estudantes, foi realizado um quase-experimento com duas turmas de $7^{\circ}$ ano, totalizando 50 alunos. Os resultados mostraram que o editor auxiliou os alunos a resolverem mais problemas no mesmo tempo.
\end{abstract}

Abstract. In this paper, we describe an intelligent editor that assists students in translating word problems into equations. PATtranslation's features help students to identify the main parts of the problem such as data, relationships and equations, as well as to access previously solved problems. It uses an hypergraph-based algorithm to represent the word problems and correct them automatically. A quasi-experiment was held in two $7^{\text {th }}$ grade classes classrooms, totaling 50 students. This evaluation showed that the editor helped students to solve more problems at the same time.

\section{Introdução}

A resolução de problemas é considerada uma das habilidades mais importantes e desafiadoras de ser desenvolvida na aprendizagem de álgebra. O conhecimento matemático ganha significado quando os alunos têm situaçõ es desafiadoras para resolver e trabalham para desenvolver estratégias de resolução (BRASIL, 1997, p. 39).

A atividade de resolução de problemas algébricos consiste de duas etapas maiores: a tradução do problema e a resolução de equaçõ es. Na fase de tradução de problemas, o estudante deve propor equaçõ es algébricas a partir de um enunciado de um problema. $\mathrm{Na}$ fase de resolução, ele deve encontrar o valor da incó gnita (geralmente, a variável $x$ ). A fase de tradução é geralmente considerada a mais difícil pelos estudantes.

Este artigo propõe um editor inteligente que assiste os estudantes na realização passo-a-passo da tradução de enunciados de problemas em equaçõ es algébricas. A ferramenta fornece funcionalidades que auxiliam o aluno a determinar qual o próximo passo a ser realizado (por exemplo, identificar partes do problema, definir relações, etc), guiando o aprendiz no seu raciocínio. Além disso, o sistema também corrige os passos realizado pelo estudante automaticamente, usando um algoritmo baseado em hipergrafos. 
O trabalho proposto visa proporcionar ao aluno a realização de todos os passos da tradução, como identificar as partes importantes do problema, definir as relaçõ es, definir as equaçõ es, além de resolver as equaçõ es através de uma integração com um sistema tutor inteligente. O sistema provê feedback ao aluno sobre a sua solução final e as soluçõ es intermediárias, além de permitir ao estudante, a qualquer momento, consultar o histó rico dos problemas resolvidos anteriormente.

Para avaliar a efetividade do sistema, foi realizado um quase-experimento com 50 estudantes de duas turmas do $7^{\circ}$ ano de uma escola particular do Rio Grande do Sul, que usaram o editor durante uma seção de laborató rio na escola, por 50 minutos. Os resultados da avaliação mostraram que o editor auxiliou os alunos a resolverem mais problemas em uma duração menor.

Este artigo encontra-se organizado como segue. A Seção 2 apresenta os conceitos sobre a resolução de problemas algébricos e a seção 3 os trabalhos relacionados. A seção 4 descreve a metodologia empregada no desenvolvimento do trabalho proposto. A seções 5 explica a avaliação realizada e seus resultados. Finalmente, a seção 6 apresenta as principais conclusões e algumas direções de trabalhos futuros.

\section{Problemas algébricos}

Os problemas algébricos apresentam uma situação ou uma histó ria que possui proposiçõ es relacionais que ligam os diversos elementos apresentados (Gama, 2004). É preciso compreender esses elementos a fim de gerar um modelo matemático para representá-los. Esse modelo consiste em uma ou mais equaçõ es. Resolver essas equaçõ es produz valores numéricos que resolvem o problema.

Um problema algébrico demanda a realização de uma sequência de ações ou operaçõ es para obter um resultado. Dessa forma, a solução não está disponível de início, mas é possível construí-la. Aprender a dar uma resposta correta pode ser suficiente para que ela seja aceita, mas não é garantia de apropriação do conhecimento envolvido. É necessário desenvolver habilidades que permitam provar os resultados, testar seus efeitos e comparar diferentes caminhos para obter a solução. Nessa forma de trabalho, a importância da resposta correta cede lugar à importância do processo de resolução (BRASIL, 1997, p. 42).

No entanto, os problemas não tem desempenhado seu verdadeiro papel no ensino, pois a prática mais frequente de sua utilização consiste em ensinar um conceito, procedimento ou técnica e posteriormente apresentar um problema para avaliar se os alunos são capazes de empregar o que lhes foi ensinado. Nesse caso, na maioria das vezes, a concepção de ensino e aprendizagem é de que o aluno aprende por imitação/repetição (BRASIL, 1997, p. 40).

Ainda assim, existem estratégias mais construtivistas de ensinar a resolução de problemas algébricos. (Polya, 2004) definiu uma série de questionamentos e sugestõ es que podem ser feitos aos alunos, com base na sua experiência em sala de aula, a fim de ajudá-los na resolução dos problemas. Seguindo os princípios de (Polya, 2004), (Gama, 2004) visionou heurísticas para a resolução do problemas, onde seus passos podem ser realizados até que 0 aluno esteja satisfeito com a solução. Essas estratégias construtivistas de ensinar são descritas nas seções a seguir. 


\subsection{Estratégias para auxiliar os estudantes a resolverem problemas algébricos}

Polya (2004) foi um professor com grande experiência no ensino da matemática, que esquematizou uma lista de perguntas e sugestõ es que podem ser feitas aos alunos repetidas vezes a fim de ajudá-los na resolução de problemas. Essas perguntas estão divididas nas fases a seguir.

Primeiramente, o aluno precisa entender a declaração verbal do problema. A partir de então, o professor pode fazer perguntas para auxiliar os estudante a identificar as partes importantes do problema: "Qual é a incó gnita? Quais são os dados? Qual é a condição?". Se é necessário dar nomes a esses objetos, o aluno deve apresentar uma notação adequada.

Segundo, o aluno deve elaborar um plano de como resolver o problema. Muita vezes, é apropriado perguntar ao aluno se ele se lembra de ter resolvido algum problema similar. Nesse caso, ele pode se basear no plano que ele usou para resolver o problema anterior.

Terceiro, o aluno deve executar o plano. A execução do plano é muito mais fácil do que a elaboração do mesmo. O plano dá uma ideia geral, e o aluno tem que examinar os detalhes um após o outro, pacientemente, até que tudo esteja perfeitamente claro.

Quarto, o aluno deve olhar para trás e examinar a soluçã o obtida. Olhando para a solução completa, considerando e reexaminando o resultado e o caminho que levou a isso, o aluno pode consolidar seu conhecimento e desenvolver suas habilidades para resolver problemas.

Finalmente, o professor deve encorajar o aluno a imaginar casos em que poderia utilizar novamente o procedimento adotado, ou aplicar o resultado obtido: "Você pode utilizar o resultado, ou o método, para algum outro problema?".

As estratégias de Polya ainda são muito empregadas para embasar trabalhos de professores e pesquisadores que desenvolvem sistemas de álgebra. Um exemplo é o sistema criado por Gama (2004).

\subsection{Heurística para tradução de problemas}

Seguindo os princípios de Polya (2004), Gama (2004) visionou algumas heurísticas para tradução de um problema matemático, que envolve a identificação e entendimento dos principais elementos: dados, objetivos, incó gnitas e relaçõ es. Os dados são os valores conhecidos e que serão utilizados para resolver o problema. Os objetivos representam o que deve ser encontrado no problema. As incó gnitas são valores não explícitos no problema, porém necessários para se chegar nos objetivos. As relaçõ es são as sentenças que conectam os dados, as incó gnitas e os objetivos. As expressõ es matemáticas das relações são as equações.

Polya e Gama acreditam que uma compreensão desses elementos pode ser um poderoso recurso que leva a uma compreensão completa do problema. Isso permite a organização do problema em um formato para o qual o aluno pode aplicar algumas estratégias de forma a traduzir o problema com sucesso em equaçõ es. Os passos básicos dessa heurística, que podem ser realizados até que o aluno esteja satisfeito com a solução, são os seguintes: 
1. Ler a descrição do problema e identificar os objetivos. O estudante deve escrever o objetivo com suas próprias palavras e criar variáveis para representá-los.

2. Ler a descrição do problema e identificar os dados. Criar uma variável para cada dado e atribuir o valor conhecido para a mesma.

3. Identificar as incó gnitas que estão explicitamente descritas no problema e definir variáveis para representá-las.

4. Selecionar, a partir da descrição do problema, as sentenças que definem relações entre dois ou mais elementos.

5. Reescrever cada relação, substituindo as partes das sentenças que se referem aos componentes por seus nomes de variáveis. Se nenhum componente previamente identificado corresponde a qualquer componente da relação, identificar seu tipo (dado, objetivo ou incógnita) e criar uma variável apropriada.

6. Analișar o significado matemático da relação e decidir quais operadores aritméticos devem ser aplicados $(+,-, *, /,=)$.

7. Traduzir a relação em uma equação, usando as variáveis e os operadores organizados na forma que melhor corresponde ao significado da sentença original.

8. Ler cada equação produzida (em mais de uma maneira, se possível), para verificar se ela traduz de volta para a sentença original.

9. Verificar cuidadosamente se alguma equação foi omitida e se todas as variáveis previamente identificadas foram utilizadas nas equações.

As vezes, o aluno apenas reconhece um aspecto importante do problema depois de algumas tentativas frustradas de gerar as equaçõ es. Nesse caso, é necessário voltar a uma etapa anterior e revê-la.

\section{Trabalhos Relacionados}

Outros trabalhos buscaram assistir estudantes na fase de tradução de problemas algébricos. O PAL Tool auxilia os alunos a organizar e representar o problema em seus componentes menores (os dados, os objetivos, as incó gnitas e relações), porém não provê feedback sobre as informaçõ es inseridas (Gama, 2004). No Cognitive Algebra Tutor, os alunos representam o problema em tabelas, gráficos e símbolos, e utilizam essas representaçõ es para responder as questõ es contidas no problema apresentado, provendo feedback e fornecendo dicas (Koedinger et al., 1997). O Ms. Lindquist provê uma conversação do sistema com o aluno, através da escolha de estratégias que levam em consideração as informaçõ es guardadas no modelo cognitivo do aluno (Heffernan and Koedinger, 2002). O Hypergraph Based Problem Solver permite aos alunos identificar as várias quantidades de um problema e apresentar expressõ es entre essas quantidades e equaçõ es entre essas expressõ es, provendo feedback para cada passo errado feito pelo aluno através de uma forma de representar internamente o problema através de hipergrafos (Arevalillo-herráez and Arnau, 2011).

Os sistemas descritos nessa seção, com exceção do Cognitive Tutor, não fornecem assistência para o aluno em todas as fases da tradução do problema. Ao contrário dos trabalhos relacionados que visam apoiar apenas alguma(s) fase(s) da tradução de problemas, o editor visa proporcionar ao aluno a realização todos os passos da tradução, como identificar as partes importantes do problema, definir as relaçõ es, definir as equaçõ es e consultar o histó rico dos problemas resolvidos anteriormente, provendo feedback em cada passo realizado. Além disso, o editor também auxilia na fase de execução da solução, através de 
uma integração com o sistema tutor inteligente PAT2Math que assiste, corrigindo cada passo e dando dicas, os estudantes na resolução passo-a-passo de equaçõ es ${ }^{1}$. Um outro aspecto positivo do editor proposto é que sua interface guia o aluno na realização dos passos: primeiramente, o aluno deve definir os dados e as incó gnitas, apó s as relaçõ es, para então propor a equação. Esse aspecto pode auxiliar a reduzir a carga cognitiva do estudante, proporcionando melhor aprendizagem.

\section{Metodologia}

Essa seção descreve a metodologia empregada no desenvolvimento do trabalho proposto. Primeiramente, foram estudadas estratégias e heurísticas para ensinar e auxiliar estudantes na fase de tradução de problemas. Foi adotada a estratégia proposta por Gama (2004), por apresentar uma sequência de passos bem definidas e estar baseada nas estratégias de Polya, que têm sido amplamente empregadas nos trabalhos relacionados. Essa estratégia apoiou inclusive o projeto da interface do PATtranslation, concebido de forma a guiar os estudantes nos passos propostos por Gama e na mesma ordem.

Numa segunda fase, foi buscado um algoritmo para corrigir automaticamente as soluçõ es dos alunos. Foi adotado o algoritmo de (Arevalillo-herráez and Arnau, 2011), que usa uma solução baseada em hipergrafos. Posteriormente, a ferramenta proposta foi implementada e integrada ao sistema tutor inteligente PAT2Math. Essa integração permite que o aluno possa resolver no tutor as equações montadas no PATtranslation.

Para avaliação, foi primeiramente realizada uma reunião com a professora de matemática das turmas envolvidas. Nessa reunião, PATtranslation foi demonstrado à professora, que sugeriu algumas pequenas modificações no layout da interface. Foi também solicitado à professora os enunciados de problemas a ser inseridos no editor para que pudessem ser trabalhados na avaliação.

Finalmente, um quase-experimento foi conduzido com duas turmas (total de 50 alunos) em uma sessão de 50 minutos de duração no laborató rio de informática da escola. Foi aplicado um pó s-teste, assim como foi registrado pelo sistema o número de problemas resolvidos corretamente por cada aluno. O teste t-student foi usado para analisar o quão significativos estatisticamente foram os resultados obtidos. As próximas seçõ es descrevem com mais detalhes essas etapas.

\section{O Editor inteligente PATtranslation}

O trabalho proposto consiste na criação de um editor inteligente, que visa auxiliar os estudantes a propor equaçõ es algébricas a partir do enunciado do problema, ou seja, na fase de tradução de problemas algébricos. O editor chama-se PATtranslation e está integrado ao sistema tutor PAT2Math (Seffrin et al., 2011), que auxilia os alunos na fase de resolução da equação. O PATtranslation é um sistema web, implementado em Java (no servidor) e HTML5 (no cliente).

\subsection{Arquitetura e comportamento}

A sua arquitetura do PATtranslation é formada por quatro componentes, integrados a arquitetura do PAT2Math: módulo do domínio, módulo do aluno, corretor e interface.

\footnotetext{
${ }^{1}$ http://pat2math.unisinos.br
} 
No mó dulo de domínio estão armazenados os dados sobre os problemas matemáticos, como seu enunciado, suas partes relevantes, suas relações, equaçõ es e os caminhos de resolução, utilizando a representação de hipergrafos. No mó dulo do aluno está armazenado o histó rico de resolução dos problemas pelos alunos, tais como os passos realizados, 0 andamento atual da resolução e o tempo investido na resolução. Os mó dulos de domínio e do aluno do PATtranslation estão integrados aos mesmos mó dulos do PAT2Math. O corretor é um algoritmo que utiliza a notação de hipergrafos, com base nos dados contidos nos outros mó dulos, a fim de fornecer o feedback do passo executado pelo aluno. A interface grá fica do editor faz a interação com o aluno e utiliza o corretor para validar os passos executados. Os problemas estão organizados em planos de aula e são inseridos por um administrador ou professor no mó dulo de domínio. O aluno pode escolher em qual ordem ele desejar resolver os problemas de um mesmo plano, no entanto, o plano seguinte apenas será disponibilizado quando o estudante tiver resolvido todos os problemas do plano atual.

\subsection{Interface}

A interface do PATtranslation, ilustrada na 1, foi projetada para permitir aos alunos definir dados, incó gnitas, relaçõ es e equaçõ es, através do enunciado de um problema matemático. No menu da esquerda, são mostrados os planos de aula, com os seus perspectivo problemas. No Tutorial, primeira opção do menu, é apresentado um vídeo explicando o funcionamento do PATtranslation.

Ao clicar em um dos problemas do menu, esse é apresentado na interface do sistema, representada como uma folha de caderno. O enunciado é mostrado no topo e os quadros menores contém campos específicos para o aluno definir as variáveis do problema, que podem ser dados, incógnita, relações e equações do problema.

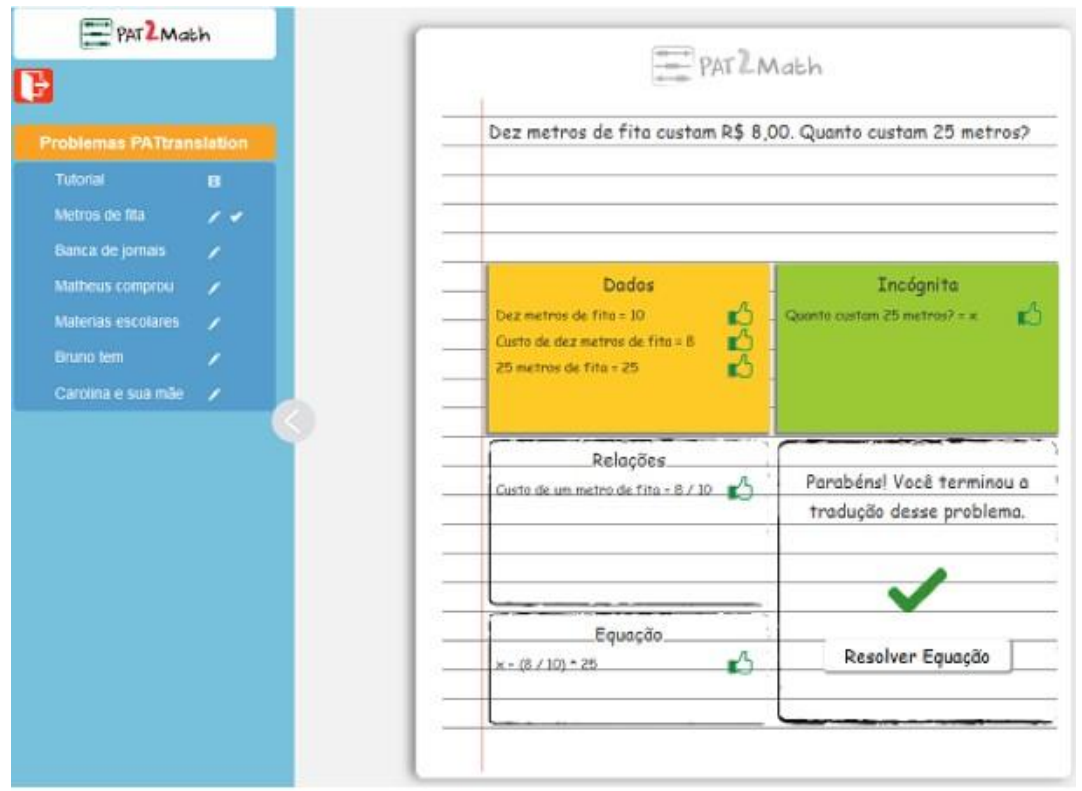

Figura 1. Interface do PATtranslation

No botõ es Dados e Incógnita, o aluno define os dados e incó gnitas do problema, respectivamente, isto é, os valores conhecidos e desconhecidos. Em ambos os casos, é 
exibida uma lista com todos os trechos do problema relacionados aos dados/incógnitas ainda não definidos e um campo onde o aluno informa o valor (no caso do dado) ou variável (para a incó gnita). Após definição de todos os dados e incó gnitas, os botões são desabilitados.

No botão Relaçõ es, o aluno define expressõ es que envolvem variáveis e/ou dados e um operador e que resultem em uma incó gnita. Ao clicar nesse botão, é mostrado ao aluno uma lista com os trechos de textos relacionados as relaçõ es a serem definidas e um campo protegido para digitação (onde o aluno monta a relação). O aluno deve montar as relações selecionando variáveis e dados definidos anteriormente e operadores.

No botão Equação, o aluno define a equação, que envolve variáveis e um operador. Após definir a equação, esse botão é desabilitado e o sistema parabeniza o aluno pela tradução correta do problema. Então, é habilitado o botão Resolver Equação, para o estudante encontrar o valor da incógnita.

\subsection{Correção passo a passo}

O PATtranslation fornece feedback para cada passo definido pelo aluno. Um feedback é um retorno mínimo visual para o aluno saber se o passo está correto (no PATtranslation ele é dado pelo símbolo de ok com a mão). No PATtranslation, um passo consiste na definição de um dado, ou incó gnita, relação ou equação. Ao definir um dado, é verificado se o valor digitado corresponde a esse dado. Se não for, o dado não é aceito e é exibida uma dica ao aluno. Ao definir uma relação ou uma equação, são consultadas as relaçõ es do problema ainda não definidas pelo aluno. Com base nas relaçõ es retornadas, é aplicado o algoritmo dos hipergrafos, descrito na seção 5.3.1. Internamente, cada uma das relaçõ es retornadas representa uma aresta do hipergrafo. Se a relação definida não corresponder a nenhuma daquelas retornadas pelo hipergrafo, a relação ou equação não será aceita. Tudo o que for definido corretamente é mostrado no seu correspondente quadro, com a imagem de uma mão com o polegar para cima (simbolizando ok), indicando que está correto.

\subsubsection{Empregando hipergrafos para correção dos passos}

Para que o sistema possa corrigir os passos do aluno, ele precisa ter alguma forma de representação do problema que lhe permita verificar se a solução do aluno está correta. Neste trabalho foi empregada a representação proposta por (Arevalillo-herráez and Arnau, 2011), que emprega hipergrafos para representar um problema algébrico. Um hipergrafo é uma generalização de um grafo no qual uma aresta pode ligar qualquer número de vértices. Quando todas as arestas têm $n$ vértices, o hipergrafo é chamado n-uniforme. Um grafo trinomial é uma representação gráfica da estrutura de um problema matemático que estende o conceito de um hipergrafo 3-uniforme, por meio de uma representação diferente para elementos conhecidos e desconhecidos. Em particular, quantidades conhecidas são representadas por vértices escuros, quantidades desconhecidas por círculos claros, e relacionamentos ternários por arestas que passam pelos três vértices envolvidos. Consequentemente, grafos trinomiais tornam possível a representação de qualquer relacionamento ternário que tem a forma $X=Y$ opZ, onde $X, Y$ e $Z$ representam quantidades e op um operador básico no conjunto $+,-, *, /$

A fim de representar as relações existentes entre as quantidades de um problema 
algébrico, o grafo trinomial foi estendido por (Arevalillo-herráez and Arnau, 2011). Arestas dirigidas são utilizadas para identificar a quantidade que fica do lado esquerdo da relação, onde no seu final existe uma seta triangular que inclui um ró tulo em seu interior para identificar a relação de forma ú nica. Ao lado da seta, existe um marcador para identificar o operador que é utilizado na relação. $O$ hipergrafo do problema matemático "O pai de Mike é três vezes mais velho que Mike. Quatro anos atrás, ele foi quatro vezes mais velho. Que idade tem o Mike?" pode ser visto na Figura 2. Os nós $F$ c e F $p$ indicam, respectivamente, a idade atual e do passado do pai de Mike. Os nós $M$ c e $M$ p indicam da mesma forma as idades de Mike (Arevalillo-herráez and Arnau, 2011).

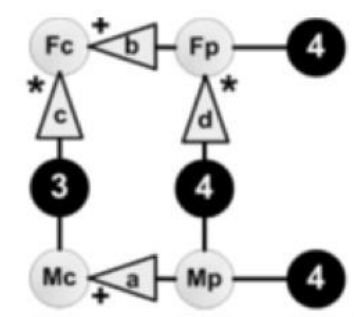

Figura 2. Exemplo de Hipergrafo para representar equações de um problema algébrico (Arevalillo-herraez Arnau, 2011)

Para ser capaz de controlar o processo de resolução, o hipergrafo é atualizado apó s cada entrada válida feita pelo aluno. $O$ aluno pode atribuir uma letra para qualquer quantidade desconhecida. O nó é então marcado como definido, e o nome da letra é atribuída a seu campo de valor. Ao apresentar uma expressão, é feita uma busca no hipergrafo, procurando um relacionamento existente que corresponde à entrada do aluno. Para tanto, são identificadas todas as arestas com exatamente um nó indeterminado. Para cada aresta identificada, é verificado se as quantidades conhecidas são as mesmas que as da expressão inserida pelo aluno. Se forem as mesmas quantidades, então é verificado se o operador escolhido e a ordem dos operandos estão corretos. Estando tudo correto, o nó indefinido na aresta fica definido, a expressão é atribuída ao seu campo de valor, e a ponta é removida do hipergrafo. Ao apresentar uma equação, é verificado se a mesma corresponde a qualquer aresta no hipergrafo que não tenha quantidade indefinida. Se alguma aresta é encontrada, a mesma é removida do hipergrafo. O problema é considerado terminado quando todas as arestas forem removidas do hipergrafo.

\section{Avaliação}

Com o objetivo de verificar se o editor auxiliou os alunos na tradução do enunciado de um problema matemático em uma equação, foi realizado um quase-experimento com duas turmas do $7^{\circ}$ ano do ensino fundamental de uma escola privada da cidade de São Leopoldo - RS. Em um quase-experimento, uma turma inteira de alunos é atribuída ao grupo de controle, enquanto outra é definida como grupo experimental. Esse tipo de avaliação é empregada quando não é possível a distribuição aleató ria na mesma turma, como no caso do trabalho proposto, pois os alunos notariam entre si que estão usando versões diferentes do sistema, o que poderia impactar nos resultados da avaliação.

O grupo experimental era uma turma composta de 24 alunos, com 12 e 13 anos de idade. O grupo de controle era formado por 26 alunos, com idades de 11 até 13 anos. 
O experimento durou 50 minutos, sendo a primeira parte o uso do PATtranslation e a segunda parte a aplicação do questionário, com duração de 35 e 15 minutos, respectivamente. Inicialmente, foi resolvido um problema de exemplo, utilizando o PATtranslation, para que os alunos entendessem o funcionamento do editor. A avaliação foi realizada no laboratório de informática da escola.

Para a avaliação, foram desenvolvidas duas versõ es do PATtranslation. Na versão do grupo de controle, é apresentado o enunciado do problema e um campo livre para o aluno entrar com a equação. Nessa versão, também não é mostrado o vídeo de Tutorial, já que a interface é muito simples. Na versão do grupo experimental, os alunos disposeram de todas as funcionalidades desenvolvidas para o PATtranslation, conforme Figura 1. A avaliação consistiu na tradução de seis enunciados de problemas algébricos. Esses problemas são dos mais variáveis tipos e foram fornecidos pela professora das duas turmas.

Durante o experimento, o sistema gravou o histó rico de açõ es do aluno no sistema. Após o uso do PATtranslation, um questionário foi aplicado a ambos grupos.

\subsection{Resultados e Discussão}

O questionário era formado por sete questõ es fechadas, cujas respostas seguiam a escala Likert. As três primeiras questõ es perguntavam sobre o quanto o aluno tinha gostado de interagir com o sistema e lhe avaliado útil. As três últimas questõ es objetivavam verificar o quanto a ferramenta tinha auxiliado o aluno a identificar as partes relevantes do problema para montar a equação. A quarta questão verificava se os estudantes buscavam estudar soluções anteriores para resolver os novos problemas.

A primeira questão do questionário buscava entender o quanto os alunos achavam interessante resolver problemas no PATtranslation. No grupo experimental, $85 \%$ alunos responderam que achavam interessante ou muito interessante, enquanto que no grupo de controle, apenas $80 \%$ dos alunos responderam afirmativamente. Quanto à pergunta que buscava verificar se os alunos preferiam resolver problemas no PATtranslation do que em papel caneta, no grupo experimental $95 \%$ responderam afirmativamente, enquanto no grupo de controle $72 \%$ dos alunos afirmaram preferir o PATtranslation. Em relação às perguntas que buscavam entender o quanto a ferramenta ajudava os estudantes a identificar as partes importantes do problema e a resolvê-lo, no grupo de controle, $71 \%$ dos alunos responderam afirmativamente, enquanto no grupo experimental $90 \%$ afirmaram que o tutor foi útil nesse aspecto.

Nos espaços reservados para expressar opiniõ es e sugestõ es, muitos alunos do grupo experimental responderam que o sistema era muito fácil e prático de usar e que haviam gostado muito de utilizar o sistema. Também foi observado que no grupo experimental os alunos precisaram interagiram muito pouco com a professora para tirar dú vidas de como traduzir os problemas.

Em relação ao nú meros de problemas resolvidos corretamente, os alunos do grupo de controle resolveram 98 problemas, enquanto os alunos do grupo experimental resolveram 129 problemas corretamente. Uma análise inicial mostra que os alunos do grupo experimental conseguiram traduzir mais problemas na mesma duração de sessão.

Para avaliar o quanto essa diferença é significativa estatisticamente, foi aplicado o 
teste $t$-student. A hipótese do experimento é a de que o PATtranslation auxilia os alunos a traduzir mais problemas algébricos corretamente, ou seja, a média de acerto dos alunos que utilizaram a versão experimental foi significativamente maior que a média dos alunos no grupo de controle. O grau de confiança adotado é de $95 \%(\alpha=0,05)$.

Aplicando o teste $t$-student, foi obtido $p=0,0018(t=-3,0615 ; d \boldsymbol{f}=45,538)$. Assim, existem evidências para afirmar com $95 \%$ de confiança que a versão completa PATtranslation auxiliou os alunos a traduzir mais problemas na mesma duração de sessão ${ }^{2}$.

\section{Conclusão}

Neste artigo, foi descrito um editor inteligente que assiste os alunos na tradução de problemas algébricos em equaçõ es. Esse editor, chamado de PATtranslation, foi integrado ao sistema tutor PAT2Math. PATtranslation permite ao aprendiz realizar todos os passos da tradução, como: identificar as partes importantes do problema, definir as relações, definir as equaçõ es, além de resolver as equaçõ es através de uma integração com o sistema tutor inteligente PAT2Math. O editor também provê feedback sobre cada passo realizado e permite consultar o histórico de problemas resolvidos anteriormente.

Foi realizado um quase-experimento do PATtranslation, a fim de verificar se os recursos propostos efetivamente auxiliaram os estudantes. Duas turmas de $7^{\mathrm{a}}$ ano do ensino fundamental utilizaram o sistema durante 50 minutos. Os resultados obtidos mostraram que a turma que utilizou a versão completa do PATtranslation traduziu mais problemas algébricos corretamente que os alunos do grupo de controle. Pretende-se realizar uma avaliação experimental com uma duração maior, de seis meses, para verificar o impacto do uso do PATtranslation na aprendizagem dos estudantes.

\section{Referências}

Arevalillo-herráez, M. and Arnau, D. (2011). A Hypergraph Based Framework for Intelligent Tutoring of Algebraic Reasoning. In Lane, H., Yacef, K., Mostow, J., and Pavlik, P., editors, Artificial Intelligence in Education, pages 512-521, Memphis, TN, USA. Springer.

BRASIL (1997). Parâmetros Curriculares Nacionais: matemática. Volume 3. Technical report, Secretária de Educação Fundamental., Brasília.

Gama, C. A. (2004). Integrating Metacognition Instruction in Interactive Learning Environments. Phd thesis, University of Sussex.

Heffernan, N. and Koedinger, K. (2002). An intelligent tutoring system incorporating a model of an experienced human tutor. Intelligent Tutoring Systems, pages 596-608.

Koedinger, K. R., Anderson, J. R., Hadley, W. H., and Mark, M. A. (1997). Intelligent tutoring goes to school in the big city. Int. J. of Artificial Intelligence in Education, 8(1):30-43.

Polya, G. (2004). How to Solve It: A New Aspect of Mathematical Method. Princeton Science Library. Princeton University Press.

Seffrin, H., Rubi, G., and Jaques, P. A. (2011). O Modelo Cognitivo do Sistema Tutor Inteligente PAT2Math. In SBIE, pages 10-19, Aracajú. SBC.

\footnotetext{
${ }^{2}$ No entanto, é importante ressaltar que essa avaliação sofre de ameaças à validade interna por não ter havido distribuição randômica dos estudantes nos grupos de controle e experimental.
}

V. $14 \mathrm{~N}^{\mathrm{o}} 1$, julho, 2016 